\title{
A NOTE ON DIV CURL INEQUALITIES
}

\author{
Loredana Lanzani And Elias M. Stein
}

Recently a series of interesting theorems have been proved by Bourgain, Brezis, Mironescu, and Van Schaftingen, [1],[2],[3],[7], that involve the divergence and curl of vector fields.

Among the many results obtained is the following surprising inequality:

Theorem A. (Bourgain and Brezis [2]). Suppose $Z$ is a smooth vector field, $Z(x)=\left(Z_{1}(x), \ldots, Z_{n}(x)\right)$, of compact support in $\mathbb{R}^{n}$, with $n \geq 3$. If curl $Z=f$ and $\operatorname{div} Z=0$, then

$$
\|Z\|_{L^{r}} \leq A\|f\|_{L^{1}} \text {, where } r=n /(n-1) .
$$

(Note: We state this result, and others below, for smooth functions or forms of compact support. More general formulations then follow by standard limiting arguments).

The result above is remarkable in view of the following facts. First, the inequality (1) fails for $n=2$. Secondly, its variant,

$$
\|Z\|_{r} \leq A\left(\|f\|_{L^{1}}+\|g\|_{L^{1}}\right),
$$

with $\operatorname{div} Z=g$, fails for every $n \geq 2$.

The inequality (1) is reminiscent of a famous inequality of Gagliardo and Nirenberg, valid for all $n \geq 1$. Here $F$ is a scalar smooth function of compact support in $\mathbb{R}^{n}$.

\section{Theorem B.}

$$
\|F\|_{L^{r}} \leq A\|\nabla F\|_{L^{1}}, r=n /(n-1) .
$$

However, it should be observed that the proof of (1) is subtler than that of (2). The question that arises is of finding a common general context for these theorems. It is the purpose of this note to help to clarify this issue by pointing out how the following result, formulated in the language of the (real) Hodge de Rham complex in $\mathbb{R}^{n}$, can be obtained by using existing techniques.

We deal with smooth $q$-forms $u$, with compact support, and denote by $d u$ and $d^{*} u$, the exterior derivative and co-exterior derivative of $u$, which are $q+1$ and $q-1$ forms, respectively. (For basic facts about the Hodge complex, see e.g. [4].) We consider the system

Received August 24, 2004.

The first author is supported by the NSF Grant No. DMS-9800794. The second author is supported by NSF Grants No. DMS-0070692 \& DMS-0245242. Both authors wish to acknowledge the kind hospitality shown them while visiting the Research Center Ennio DeGiorgi in Pisa. 


$$
\left\{\begin{array}{l}
d u=f \\
d^{*} u=g
\end{array} .\right.
$$

We then ask if an inequality of the form

$$
\|u\|_{L^{r}} \leq A\left(\|f\|_{L^{1}}+\|g\|_{L^{1}}\right), \quad r=n /(n-1)
$$

is valid, and if not, what are appropriate substitutes.

Theorem. If $u$ is a smooth $q$-form with compact support on $\mathbb{R}^{n}$ then:

(a) The inequality (3) holds when $q$ is neither 1 nor $n-1$.

(b) When $q=1$ a substitute for (3) holds with $\|g\|_{L^{1}}$ replaced by $\|g\|_{H^{1}}$, with $H^{1}$ the real Hardy space. Similarly for $q=n-1$, when we replace $\|f\|_{L^{1}}$ by $\|f\|_{H^{1}}$.

We should note first that the case $q=0, q=n$ are merely restatements of Theorem B, while the case $q=1$, when $g=0$, is Theorem A. We should add that we have been informed by Bourgain and Brezis that the above theorem can also be deduced from general results of theirs, contained in a forthcoming paper.

While there are several approaches possible to the proof of this theorem, the one we outline is an adaptation of an argument in [7]. It is based on the following estimate. We have stated this in a sharper form than needed in the present case, because the stronger inequality may turn out to be useful later.

On $\mathbb{R}^{N}$, besides the spaces $L^{p}\left(\mathbb{R}^{N}\right)$, we need the corresponding "weak-type" space of functions $F$, those for which $m\{x:|F(x)|>\alpha\} \leq A / \alpha^{p}$ for all $\alpha>0$. For any $F$ we denote by $F^{*}$ the equi-measurable decreasing rearrangement of $|F|$ on $(0, \infty)$. Then $F$ satisfies this weak-type inequality when the quantity sup $t^{1 / p} F^{*}(t)$ is finite, and if $1<p<\infty$, this quantity is equivalent to a norm on the corresponding Lorentz space $L^{p, \infty}$. See e.g. [6], Chapter V, §3.

Next, on $\mathbb{R}^{n}$ we define a space of functions that are of weak-type in all but one variable. More precisely for $x \in \mathbb{R}^{n}$ write $x$ as $\left(x_{1}, \bar{x}\right), x_{1} \in \mathbb{R}, \bar{x} \in \mathbb{R}^{N}$, $N=n-1$, and define the space $\widetilde{L}^{p}$ in terms of its norm by

$$
\|F\|_{L^{p}}=\left(\int_{\mathbb{R}^{1}}\left\|F\left(x_{1}, \cdot\right)\right\|_{L^{p, \infty}\left(\mathbb{R}^{n-1}\right)}^{p} d x_{1}\right)^{1 / p} .
$$

One observes that $\|F\|_{L^{p}} \leq c\|F\|_{L^{p}}$, so $L^{p} \subset \widetilde{L}^{p}$, but the inclusion is strict.

Lemma 1. Suppose $F_{1}, F_{2}, \ldots F_{n}$ are smooth functions of compact support with $\frac{\partial F_{1}}{\partial x_{1}}=\sum_{k=2}^{n} \frac{\partial F_{k}}{\partial x_{k}}$, then

$$
\left|\int_{\mathbb{R}^{n}} F_{1}(x) \Phi(x) d x\right| \leq c\left(\sum_{k=1}^{n}\left\|F_{k}\right\|_{L^{1}}\right)\|\nabla \Phi\|_{L^{p}}
$$


This is based on the following.

$$
\text { with } \quad p=n \text {. }
$$

Lemma 2. Suppose $\Phi$ is smooth and has compact support in $\mathbb{R}^{N}$. Then for every $\delta>0$ we can write $\Phi=\Phi_{1}+\Phi_{2}$ with

$$
\sup _{x \in \mathbb{R}^{N}}\left|\Phi_{1}(x)\right| \delta^{-\gamma}+\sup _{x \in \mathbb{R}^{N}}\left|\nabla \Phi_{2}(x)\right| \delta^{1-\gamma} \leq c\|\nabla \Phi\|_{L^{p, \infty}\left(\mathbb{R}^{N}\right)},
$$

as long as $p>N$ and $\gamma=1-N / p$.

To prove Lemma 2, recall that $\Phi=K * \nabla \Phi$, with $K=c_{N} x /|x|^{N}$. Decompose $K$ as $K_{1}+K_{2}$, where $K_{1}(x)=K(x) \eta(x / \delta)$, with $\eta$ a smooth cut-off function which is 1 when $|x| \leq 1 / 2$, and is supported in $|x| \leq 1$. We then set $\Phi_{j}=K_{j} * \nabla \Phi$, $j=1,2$, and use the basic rearrangement inequality $\int_{\mathbb{R}^{N}}|F(x)||G(x)| d x \leq$ $\int_{0}^{\infty} F^{*}(t) G^{*}(t) d t$. First we set $F=K_{1}$ and $G=\nabla \Phi$. A calculation shows that $F^{*}(t) \leq c t^{-\frac{N-1}{N}}$, for $0 \leq t \leq c_{1} \delta^{N}$, and $F^{*}(t)=0$, otherwise. Hence,

$$
\left|\Phi_{1}\right| \leq c^{\prime} \int_{0}^{c_{1} \delta^{N}} t^{-\frac{N-1}{N}} t^{-/ p}\|\nabla \Phi\|_{L^{p, \infty}} d t=c \delta^{\gamma}\|\nabla \Phi\|_{L^{p, \infty}} .
$$

Similarly, $\nabla \Phi_{2}=\nabla K_{2} * \nabla \Phi$. With $F=\nabla K_{2}$, another calculation shows that $F^{*}(t) \leq c\left(t+c_{1} \delta^{N}\right)^{-1}$. Thus one has $\left|\nabla \Phi_{2}\right| \leq c \delta^{-1+\gamma}\|\nabla \Phi\|_{L^{p, \infty}}$, because $\int_{0}^{\infty}\left(t+c_{1} \delta^{N}\right)^{-1} t^{-1 / p} d t=\bar{c} \delta^{-1+\gamma}$. This gives Lemma 2 .

To prove Lemma 1, one can now follow the argument in Van Schaftingen [7]. For each function $F$ on $\mathbb{R}^{n}$ we write $F^{y}$ for the slice on $\mathbb{R}^{n-1}$ obtained by freezing the $x_{1}$ variable as $y$, that is $F^{y}(\bar{x})=F(y, \bar{x})$.

We set $J(y)=\int_{\mathbb{R}^{n-1}} F_{1}^{y}(\bar{x}) \Phi^{y}(\bar{x}) d \bar{x}$. Then using the decomposition of Lemma 2 for each $y$, with $N=n-1$, we get that $J(y)=J_{1}(y)+J_{2}(y)$, where $J_{j}(y)$ is obtained from $J(y)$ by replacing $\Phi^{y}$ by $\Phi_{j}^{y}$. Thus we obtain

$$
\left|J_{1}(y)\right| \leq c \delta^{\gamma}\left\|F_{1}^{y}\right\|_{L^{1}\left(\mathbb{R}^{N}\right)}\left\|\nabla \Phi^{y}\right\|_{L^{p, \infty}\left(\mathbb{R}^{N}\right)} .
$$

Also $J_{2}(y)$

$$
\begin{aligned}
&=\int_{-\infty}^{y} \int_{\mathbb{R}^{N}} \frac{\partial}{\partial x_{1}}(\left.F_{1}\left(x_{1}, \bar{x}\right)\right) \Phi_{2}^{y}(\bar{x}) d x_{1} d \bar{x} \\
&=\sum_{k=2}^{n} \int_{-\infty}^{y} \int_{\mathbb{R}^{N}} \frac{\partial F_{k}}{\partial x_{k}}\left(x_{1}, \bar{x}\right) \Phi_{2}^{y}(\bar{x}) d x_{1} d \bar{x} \\
&=-\sum_{k=2}^{n} \int_{-\infty}^{y} \int_{\mathbb{R}^{N}} F_{k}(x, \bar{x}) \frac{\partial}{\partial x_{k}} \Phi_{2}^{y}(\bar{x}) d x_{1} d \bar{x} .
\end{aligned}
$$


This gives $\left|J_{2}(y)\right| \leq c \delta^{-1+\gamma}\left(\sum_{k=1}^{n}\left\|F_{k}\right\|_{L^{1}\left(\mathbb{R}^{n}\right)}\right)\left\|\nabla \Phi^{y}\right\|_{L^{p, \infty}\left(\mathbb{R}^{n}\right)}$. We then combine these estimates, choosing $\delta=\delta(y)=\left\|F_{1}^{y}\right\|_{L^{1}\left(\mathbb{R}^{n}\right)}^{-1} \cdot \sum_{k=1}^{n}\left\|F_{k}\right\|_{L^{1}\left(\mathbb{R}^{n}\right)}$ and integrate in $y$, using Hölder's inequality (with exponents $r=\frac{n-1}{n}$ and $p=n$ ) and the fact that $\gamma=1-N / p=1-\frac{n-1}{n}$.

We come to the proof of the theorem, and first recall some relevant notation. A $q$-form $u$ on $\mathbb{R}^{n}$ can be written in standard coordinates $u=\sum_{|I|=q} u_{I} d x^{I}$. Here $u_{I}$ are scalar functions; the $I$ 's range over multi-index sets of degree $q$, that is, each $I$ is of the form $\left\{i_{1}<i_{2}<\cdots<i_{q}\right\}$, with $1 \leq i_{j} \leq n$, and $|I|$ designates the degree of $q$; also $d x^{I}$ is an abbreviation for $d x_{i_{1}} \wedge d x_{i_{2}} \cdots \wedge d x_{i_{q}}$. If $v$ is another $q$-form, $v=\sum_{|I|=q} v_{I} d x^{I}$, then the inner product $(u, v)$ is defined as $\sum_{|I|=q} \int_{\mathbb{R}^{n}} u_{I}(x) v_{I}(x) d x$. We also note that if $u$ and $v$ are smooth forms of degrees $q$ and $q+1$ respectively, and of compact support, we have $(d u, v)=\left(u, d^{*} v\right)$. We write $\|u\|_{L^{r}}$ for $\sum_{|I|=q}\left\|u_{I}\right\|_{L^{r}}$, whenever $u$ is a $q$-form.

Now to prove the inequality (3) it suffices to prove that

$$
|(u, \varphi)| \leq A\left(\|f\|_{L^{1}}+\|g\|_{L^{1}}\right)\|\varphi\|_{L^{p}}, \text { with } p=n-1,
$$

whenever $\varphi$ is a smooth $q$-form of compact support and $d u=f, d^{*} u=g$. Assume first that $2 \leq q \leq n-2$. Let $\mathcal{G}$ be the usual fundamental solution operator for $\triangle$ on $\mathbb{R}^{n}$. Then since $\triangle=d^{*} d+d d^{*}$, we have have that

$$
(u, \varphi)=\left(u,\left(d^{*} d+d d^{*}\right) \mathcal{G}(\varphi)\right)=(d u, d \mathcal{G}(\varphi))+\left(d^{*} u, d^{*} \mathcal{G}(\varphi)\right) .
$$

Now $(d u, d \mathcal{G}(\varphi))=(f, \Phi)$, with $f$ and $\Phi$ the $q+1$ forms given respectively as $f=d u, \Phi=d \mathcal{G}(\varphi)$. Thus, to estimate $(d u, d \mathcal{G}(\varphi))$ it suffices to estimate $\int_{\mathbb{R}^{n}} f_{I}(x) \Phi_{I}(x) d x$, for each multi-index $I$ of degree $q+1$.

However since $q \leq n-2, q+1 \leq n-1$, there is an index $i, 1 \leq i \leq n$, with $i \notin I$. To simplify the notation we may relabel the indices $1,2, \cdots, n$, so that $i=1$. Next for each $k, k \neq 1$, define $I_{k}$ to be the multi-index of degree $q+1$ obtained from $I$ by adding the index 1 , but removing the index $k$, if $k \in I$. (Otherwise, if $k \notin I$, leave $I_{k}$ as undefined.) Now $d f=0$, since $d u=f$, and as a result

$$
\frac{\partial f_{I}}{\partial x_{1}}=\sum_{k=2}^{n} \pm \frac{\partial f_{I_{k}}}{\partial x_{k}}
$$


for an appropriate choice of \pm signs, where it is understood that if $k \notin I, k \geq 2$, the corresponding term in the sum does not appear. Hence by Lemma 1, with $F_{1}=f_{I}, F_{k}= \pm f_{I_{k}}$, we get

$$
\begin{aligned}
& \left|\int f_{I} \Phi_{I} d x\right| \leq c\|f\|_{L^{1}}\|\nabla \Phi\|_{L^{p}} \leq c\|f\|_{L^{1}}\|\nabla \Phi\|_{L^{p}} \\
& \quad \leq c\|f\|_{L^{1}}\|\varphi\|_{L^{p}}, \text { since }\|\nabla \Phi\|_{L^{p}}=\|\nabla d \mathcal{G}(\varphi)\|_{L^{p}} .
\end{aligned}
$$

A similar estimate works for $\left(d^{*} u, d^{*} \mathcal{G}(\varphi)\right)$ and $(5)$ is proved and thus also (3). In the case $q=0$ the term $\left(d^{*} u, d^{*} \mathcal{G}(\varphi)\right)$ is not present, and we proceed as before. Similarly for $q=n$. Finally when $q=1$, Lemma 2 does not apply to the term $\left(d^{*} u, d^{*} \mathcal{G}(\varphi)\right)$. We use instead the fact that with $\varphi=\sum_{k=1}^{n} \varphi_{k}(x) d x_{k}$

$$
\left(d^{*} u, d^{*} \mathcal{G}(\varphi)\right)=-\left(g, \sum_{k<1}^{n} \frac{\partial}{\partial x_{k}} \mathcal{G}\left(\varphi_{k}\right)\right)=\left(I_{1}(g), \sum_{k=1}^{n} R_{k}\left(\varphi_{k}\right)\right),
$$

where $R_{k}$ are the Riesz transforms, and $I_{1}$ maps $H^{1}$ to $L^{r}, r=\frac{n-1}{n}$. (See e.g. Stein [5], p.136). This gives $|(u, \varphi)| \leq c\left(\|f\|_{L^{1}}+\|g\|_{H^{1}}\right)\|\varphi\|_{L^{p}}$, and proves that $\|u\|_{L^{r}} \leq c\left(\|f\|_{L^{1}}+\|g\|_{H^{1}}\right)$, when $q=1$. A similar argument works in the case $q=n-1$, and this completes the proof of the theorem.

\section{References}

[1] J. Bourgain and H. Brezis, On the equation div $Y=f$ and application to control of phases, J. Amer. Math. Soc., 16, (2003), 393-426.

[2] New estimates for the Laplacian, the div-curl, and related Hodge systems, C. R. Math. Acad. Sci. Paris, 338, (2004), 539-543.

[3] J. Bourgain, H. Brezis, and D. Mironescu, $H^{1 / 2}$ maps with values into the circle; minimal connections, lifting and the Ginzburg-Landau equation, Publ. Math. Inst. Hautes Études Sci., (to appear).

[4] G. Schwarz, Hodge decomposition - a method for solving boundary value problems, Lecture Notes in Math, 1607, Springer-Verlag, Berlin, 1995.

[5] E. M. Stein, Harmonic Analysis, Princeton University Press, 1993.

[6] E. M. Stein and G. Weiss, Introduction to Fourier analysis on Euclidean spaces, Princeton University Press, 1971.

[7] J. Van Schaftingen, Estimates for $L^{1}$ vector fields, C. R. Math. Acad. Sci. Paris 339 (2004), 181-186.

Department of Mathematics, University of Arkansas, Fayetteville, AR 72701

E-mail address: lanzani@uark.edu

Department of Mathematics, Princeton University, Princeton, NJ 08544

E-mail address: stein@math.princeton.edu 